\title{
Biotin-Responsive Basal Ganglia Disease: Neuroimaging Features before and after Treatment
}

\author{
H. Kassem, A. Wafaie, S. Alsuhibani, and T. Farid
}

\begin{abstract}
BACKGROUND AND PURPOSE: Biotin-responsive basal ganglia disease is an autosomal recessive neurometabolic disorder presenting with subacute encephalopathy that can cause death if left untreated. The purpose of this study is to assess the neuroimaging and clinical features of the disease before and after treatment with biotin.
\end{abstract}

MATERIALS AND METHODS: We retrospectively reviewed the clinical, laboratory, and neuroimaging features of 15 genetically-proved Middle Eastern cases of biotin-responsive basal ganglia disease. Brain MR imaging was done at the onset of symptoms in all cases and within 2-8 weeks after biotin and thiamine therapy in 14 patients. The MR imaging datasets were analyzed according to lesion location, extent, and distribution.

RESULTS: Brain MR imaging showed bilateral lesions in the caudate nuclei with complete or partial involvement of the putamen and sparing of the globus pallidus in all cases. In $80 \%$, discrete abnormal signals were observed in the mesencephalon, cerebral corticalsubcortical regions, and thalami. In 53\%, when the disease was advanced, patchy deep white matter affection was found. The cerebellum was involved in $13.3 \%$. The signal abnormality of the mesencephalon, cortex, and white matter disappeared after treatment whereas the caudate and putamen necrosis persisted in all patients, including those who became asymptomatic.

CONCLUSIONS: Biotin-responsive basal ganglia disease is a treatable underdiagnosed disease. It should be suspected in pediatric patients with unexplained encephalopathy whose brain MR imaging shows bilateral and symmetric lesions in the caudate heads and putamen, with or without involvement of mesencephalon, thalami, and cortical-subcortical regions, as the therapeutic trial of biotin and thiamine can be lifesaving.

ABBREVIATIONS: BBGD = biotin-responsive basal ganglia disease; MELAS = mitochondrial myopathy, encephalopathy, lactic acidosis, and strokelike episodes; MERRF = myoclonic epilepsy associated with ragged red fibers; $\mathrm{WE}=$ Wernicke encephalopathy

B iotin-responsive basal ganglia disease (BBGD) was first described by Ozand et al in 10 patients of Arab ancestry in $1998 .^{1}$ Subsequently, cases have been reported in patients of different ethnicities including those of Portuguese, Indian, Japanese, and German origin. ${ }^{2-6}$

$\mathrm{BBGD}$ is an autosomal recessive neurometabolic disorder. It is

Received December 2, 2013; accepted after revision March 14, 2014.

From the Department of Radiology (H.K.), Benha University, Benha, Egypt; Department of Radiology (H.K.), Prince Sultan Military Medical City, Riyadh, Saudi Arabia; Department of Radiology (A.W.), Cairo University, Cairo, Egypt; University of Dammam (S.A.), Dammam, Saudi Arabia; and Pediatric Department of the Egyptian National Research Center (T.F.), Giza, Egypt.

Paper previously presented as a scientific formal oral presentation (SSQ 17-05) at: Scientific Assembly and Annual Meeting of the Radiological Society of North America, December 5, 2013; Chicago, Illinois.

Please address correspondence to Ahmed Wafaie MD, FRCR, Professor of Radiology, Faculty of Medicine, Cairo University, 7 Shaheen St, Al-Agouza, Giza, Egypt

12311; e-mail: a_wafaie@yahoo.com

三 indicates article with supplemental on-line table.

http://dx.doi.org/10.3174/ajnr.A3966 characterized by subacute encephalopathy with confusion, seizure, dysarthria, and dystonia following a history of febrile illness. If left untreated with biotin, the disease can progress to severe quadriparesis and even death. $^{7}$

Biotin is a water-soluble vitamin belonging to the $\mathrm{B}$ complex and acts as a co-enzyme for the 4 important carboxylases in humans. ${ }^{3}$ Biotin-dependent carboxylases catalyze the fixation of bicarbonate in organic acids and play crucial roles in the metabolism of fatty acids, amino acids, and glucose. The carboxylase activities decrease substantially when there is biotin deficiency. ${ }^{8}$ The etiology of biotin deficiency is believed to be caused by a genetic defect in the transporter system of biotin across the bloodbrain barrier. ${ }^{1}$ In 2005, it was demonstrated that homozygous mutation in SLC19A3 is the genetic defect that causes BBGD. ${ }^{9}$

$B B G D$ responds to the administration of high doses of biotin, which ensures some biotin transport into brain by nonspecific diffusion. ${ }^{10}$ It also increases the expression of the SLC19A3 gene, thus restoring some function of the mutated receptor. ${ }^{11,12} \mathrm{Re}-$ cently, thiamine has been added to biotin in the treatment regi- 


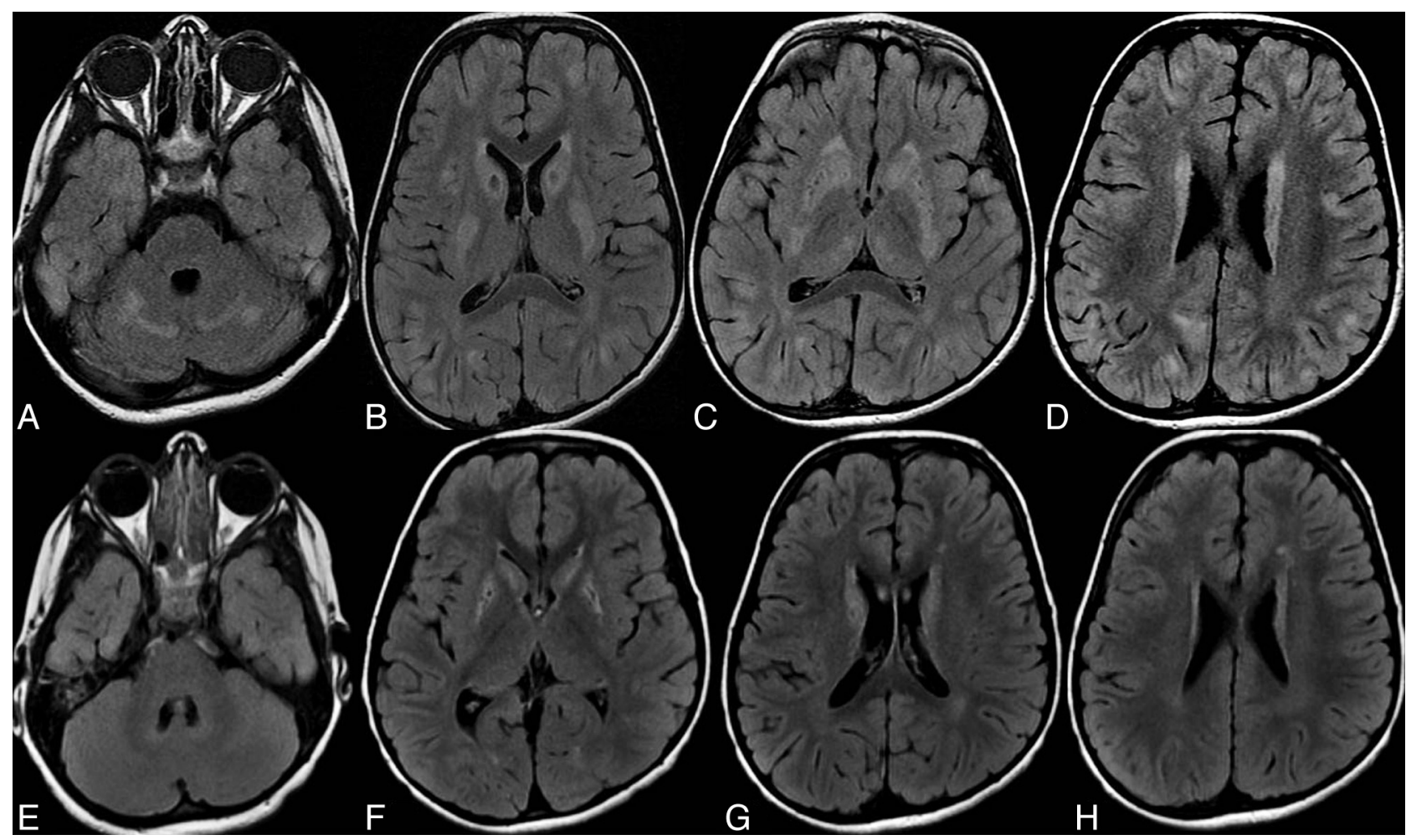

FIG 1. MR imaging of the brain in a male patient, 4 years old, with subacute encephalopathy. Axial FLAIR before $(A-D)$ and 4 weeks after $(E-H)$ treatment with biotin and thiamine. $A$, Abnormal areas of increased signal intensity in the cerebellum. $B$ and $C$, Abnormal high signal and swelling of the caudate nuclei showing small central focal necrosis with involvement of putamina and medial thalami. $D$, Focal high cortical and subcortical signals at both frontal and parietal lobes. Follow-up MR imaging after treatment $(E-H)$ shows resolution of the abnormal signal in the cerebellar, cortical, and subcortical areas. The caudate nuclei and putamina are evolved from swelling into atrophy and necrosis with persistent abnormal signal intensity.

men of BBGD based on the hypothesis that the impairment of thiamine transport in the brain also has a critical role in this disorder. This combination of biotin and thiamine proved to prevent the recurrence of encephalopathy crisis. ${ }^{7,9}$

It is important to recognize this disease because its symptoms may be reversed and the progression of its clinical course can be prevented simply by the administration of biotin and thiamine. ${ }^{1,7}$

The purpose of this study is to assess the clinical and neuroimaging features of the disease on MR imaging before and after treatment with biotin and thiamine.

\section{MATERIALS AND METHODS}

We retrospectively reviewed the clinical, laboratory, and neuroimaging features of 15 genetically proved cases of biotin-responsive basal ganglia disease. The patients were 9 females and 6 males with an age range (at the time of symptom onset) of 2 to 11 years (mean: 6 years). MR imaging of the brain was done in all cases at the onset of symptoms. Follow-up MR imaging within 2-8 weeks after the administration of high doses of biotin $(5-10 \mathrm{mg} / \mathrm{kg} / \mathrm{d})$ and thiamine $(100-300 \mathrm{mg} / \mathrm{d})$ was done in 14 patients.

All patients were of Arab (Middle East) ancestry, and 73\% had consanguineous parents. Recessive genetic defect was detected in all cases. All cases were found to have the same homozygous mutation in exon 5 of the SLC19A3 gene and their parents were heterozygous for this mutation. The molecular genetic analysis of the SLC19A3 gene was performed in the Center for Human Genetics (Bioscientia, Ingelheim, Germany). The blood and CSF chemical tests for organic and inorganic toxic compounds, carboxylases, and biotinidase values were normal in all patients.

All patients presented with subacute or acute encephalopathy leading to seizures, extrapyramidal symptoms, quadriparesis, or quadriplegia and coma.

The MR imaging was performed in all patients using the Signa LX 1.5T machine (GE Healthcare, Milwaukee, Wisconsin). Our brain MR imaging protocol included sagittal spin-echo T1WI (TR 535 milliseconds, TE 13 milliseconds), axial and coronal FSE T2WI (TR 3500 milliseconds, TE 182 milliseconds), axial FLAIR (TR 9000 milliseconds, TI 2500 milliseconds, TE 104 milliseconds), axial spin-echo T1WI (TR 535 milliseconds, TE 13 milliseconds), and axial DWI $(b=0-1000)$. Postgadolinium axial and coronal T1WI were performed in 9 cases.

Spectroscopy $\left({ }^{1} \mathrm{H}-\mathrm{MR}\right)$ was performed in 6 cases at the time of onset of symptoms and before starting the biotin therapy. After therapy MR spectroscopy was done in only 1 of these 6 cases. MR spectroscopy was performed through point-resolved proton spectroscopy sequences with TE of 35 and $144 \mathrm{msec}$. The voxels were positioned in axial T2WI on areas showing an abnormal signal at the basal ganglia and parieto-occipital cortical-subcortical regions of either the left or right side. Voxels were $2 \times 2 \times 2 \mathrm{~cm}$ in size. NAA was assigned at 2.02 parts per million, Cho at $3.2 \mathrm{ppm}, \mathrm{Cr}$ at 3.03 $\mathrm{ppm}$, and lactate at $1.3 \mathrm{ppm}$. Metabolite ratios (NAA/Cr and Cho/ $\mathrm{Cr}$ ) were also measured. All data processing was performed by software provided by the scanner manufacturer. 


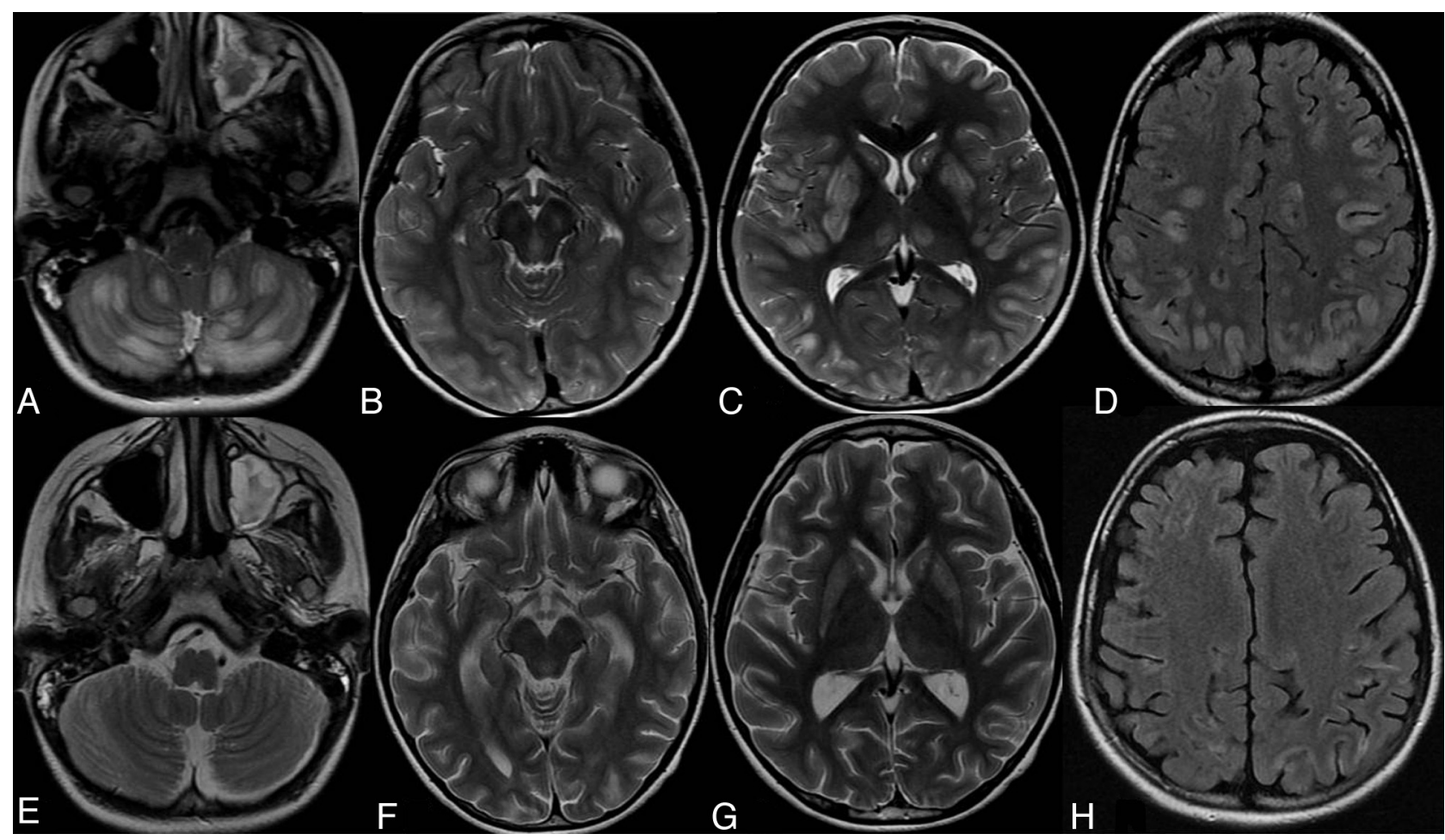

FIG 2. Brain MR imaging of a female patient, 8 years old, presenting with subacute encephalopathy and focal seizures. Axial T2WI $(A-C)$ and FLAIR $(D)$ before and 3 weeks after $(E-H)$ treatment with biotin and thiamine. A, Abnormal areas of increased signal intensity in the cerebellum with involvement of the dentate nuclei. $B$, Increased signal intensity in the mesencephalon involving the red nuclei and periaquiductal gray matter. C, Swelling and increased signal intensity in the caudate nuclei, putamina, and medial thalami with small focal necrosis in the caudate heads. $D$, Widespread focal cortical and subcortical high signal areas at both cerebral hemispheres (could represent transient edema attributed to recent seizures and not necessarily the disease). Follow-up MR imaging after treatment $(E-H)$ shows resolution of the cerebellar and mesencephalic lesions with notable regression of the thalamic, cortical, and subcortical lesions. The caudate nuclei and putamina show persistent signal abnormality and have evolved from swelling into atrophy.

Spinal cord MR imaging was done in 7 cases. The MR imaging spine protocol included sagittal T1WI (TR 397 milliseconds, TE 12 milliseconds) and T2WI (TR 3700 milliseconds, TE 103 milliseconds). Axial T2WI (TR 4220 milliseconds, TE 101 milliseconds) was performed at cervical and dorsal levels.

\section{Image Analysis}

MR examinations of the brain and spine and MR spectroscopy datasets were reviewed by 2 experienced radiologists in consensus with an emphasis on the involvement or sparing of the mesencephalon, cerebellum, caudate nuclei, globus pallidi, putamina, thalami, cortical-subcortical regions, and deep white matter before and after treatment as well as the presence of lactate doublet, NAA peak, and NAA/Cr ratio on MR spectroscopy.

The study was approved by the ethical committees of our institutions.

\section{RESULTS}

Our study enrolled 15 patients (their clinical and neuroimaging data are shown in the On-line Table). Eleven cases (73\%) had positive consanguinity. On presentation, 13 patients $(86.7 \%)$ had subacute onset in the form of encephalopathy, ataxia, and seizures, whereas only 2 patients (13.3\%) presented by acute crisis with severe neurologic deficit, generalized dystonia, quadriplegia, and coma. Pre-existing trigger factor (febrile illness or trauma) was found in 7 cases $(46.7 \%)$.
The brain MR imaging showed bilateral and symmetric lesions in the caudate heads in all of our patients with complete or partial involvement of the putamen (Figs 1-4). The globus pallidi were spared in all patients. In 12 cases (80\%), discrete abnormal signal changes were observed in the mesencephalon (Fig 2), cortical-subcortical regions, and the medial dorsal nuclei of the thalami (Figs 1-3). In 8 cases (53\%), when the disease was advanced, patchy deep white matter affection was found. The cerebellum along the cerebellar cortex and vermis was involved in only 2 patients (13.3\%) (Figs 1 and 2). The affected brain regions showed variable swelling and vasogenic edema during the acute/subacute phase. No cytotoxic edema was found. No pattern of diffusion restriction on diffusion-weighted images or significant contrast enhancement was detected.

The MR imaging of patients under treatment showed evolution of the basal ganglia from swelling into atrophy and necrosis in all cases with resolution of the abnormal high signals of the cortex and subcortical regions. Resolution of the cerebellar abnormal signal was also observed in the 2 patients who showed cerebellar involvement. However, the abnormal signals of the caudate and putamen persisted in all patients (Figs 1-4).

Pretherapy ${ }^{1} \mathrm{H}-\mathrm{MR}$ spectroscopy showed consistent elevation of lactate within the affected regions and decrease in NAA peak and NAA/Cr ratio in all cases. Disappearance of the lactate peak after biotin/thiamine therapy was noted in the single obtained posttherapy MR spectroscopy (Fig 4). 


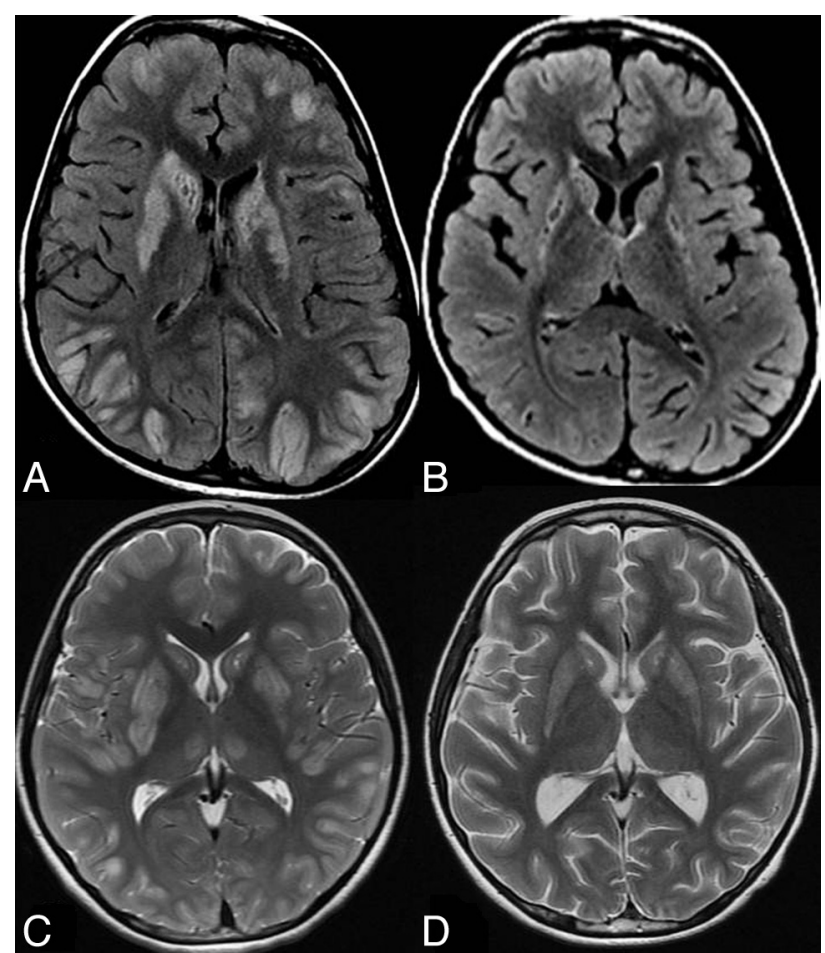

FIG 3. Brain MR imaging in 2 different patients with BBGD ( $A$ and $B$, axial FLAIR for patient 1; $C$ and $D$, axial T2WI for patient 2). In the 2 patients, the pretreatment images $(A, C)$ demonstrate bilateral swelling and increased signal in the caudate nuclei and putamina with abnormal signal in the cortex and gray-white matter junction at multiple locations. Involvement of the medial thalami is also noted in patient 2 (C). Follow-up after treatment $(B, D)$ shows disappearance of the abnormal signal in the cortical and subcortical lesions with resolution of the thalamic lesions in patient 2, who developed mild brain atrophy. The basal ganglia became atrophic with persistent abnormal signal intensity.

The spinal MR imaging done for 7 cases at the onset of symptoms showed normal appearance of the spinal cord.

All patients received a combination of biotin and thiamine. Twelve cases ( $80 \%$ ) had a good (favorable) outcome with no clinical sequelae. Two patients $(13.3 \%)$ had mild to moderate sequelae (unfavorable outcome) in the form of dystonia and dysarthria that did not interefere with daily life or school activities. One patient $(6.7 \%)$ died from severe encephalopathy, coma, cardiac arrest, and rhabdomyolysis. The high signal abnormality of the mesencephalon and cerebral/cerebellar cortical and subcortical areas disappeared after treatment whereas the caudate and putamen necrosis persisted in the 14 cases that underwent follow-up.

\section{DISCUSSION}

BBGD is an autosomal recessive neurometabolic disorder. ${ }^{7}$ It was first described in 1998 in 10 patients from the Middle East. ${ }^{1}$ Recently, the disease was recognized as a panethnic condition that usually occurs in preschool and school-aged children. ${ }^{2,4}$

The typical clinical presentation of patients with BBGD is recurrent subacute episodes of encephalopathy, often triggered by febrile illness or mild trauma and characterized by confusion, seizures, dystonia, external ophthalmoplegia, and dysphagia, eventually leading to coma and even death. Less frequently, patients with BBGD present with a chronic or slowly progressive condition characterized by dystonia, seizures, and psychomotor delay. $^{13}$
The neurologic history of most our patients (86.7\%) was in the form of subacute encephalopathy similar to the first 10 patients described by Ozand et al. ${ }^{1}$ However, 2 patients (13.3\%) presented with acute crisis with severe neurologic deficit, generalized dystonia, quadriplegia, and coma. A pre-existing trigger factor (febrile illness or trauma) was found in $46.7 \%$ of our cases.

Early diagnosis of BBGD is crucial because early administration of biotin and thiamine results in partial or complete improvement within days. Delay or lack of treatment may result in death or severe neurologic sequelae, including dystonia and quadriparesis. Moreover, patients require counseling regarding life-long treatment. ${ }^{13}$

In the original report by $\mathrm{Ozand}$ et $\mathrm{al},{ }^{1}$ the $\mathrm{MR}$ imaging findings consisted of bilateral necrosis in the basal ganglia, particularly at the central part of the caudate heads and part or all of the putamen with severe edema during the acute/subacute crisis in addition to white matter involvement at the gray-white matter junction. Subsequent reports confirmed these findings and added the involvement of the thalami, cerebellum, and brain stem. ${ }^{2,5}$ Our results supported the findings of these previous reports regarding the MR imaging distribution of lesions in all cases. In addition, the globus pallidi were spared in all patients in agreement with most of previous reports. ${ }^{1,2,13}$

Alfadhel et $\mathrm{al}^{7}$ emphasized diffuse involvement of the cortical and subcortical white matter in the acute phase and atrophy and necrosis of the basal ganglia in the chronic phase in all cases. The thalami and brain stem were involved in one-third of their patients. In the present study, we demonstrated discrete abnormal signal changes in the mesencephalon, cerebral cortical-subcortical regions, and in the medial dorsal nuclei of the thalami in $80 \%$ of cases. In 53\%, when the disease was advanced, patchy deep white matter affection was found. The cerebellum along the cerebellar cortex and vermis was involved in only $13.3 \%$ of our cases compared with one-third of the patients of Alfadhel et al. ${ }^{7}$

Follow-up of our patients under treatment confirmed the evolution of the basal ganglia from swelling into atrophy and necrosis with resolution of the abnormal high signals of the cerebral cortex and subcortical areas together with regression of the cerebellar abnormal signal in patients who showed cerebellar involvement. However, the abnormal signal lesions of the caudate and putamen persisted. These findings are in absolute agreement with earlier reports. ${ }^{1-4,7,13}$

Despite the extensive abnormal findings observed on the brain MR imaging, $80 \%$ of our patients with biotin-responsive encephalopathy remained asymptomatic under treatment. This observation suggests that enough tissue may be preserved in the target areas of the disease, to permit neurologic function. ${ }^{1}$

Interestingly, in the report by Alfadhel et al, ${ }^{7} 1$ patient demonstrated spinal cord involvement with increased T2 signal intensity particularly at the cervical region, which was not reported on earlier studies. They thought that the spinal cord involvement could be part of the disease and advised performing spinal MR imaging for all patients with BBGD. However, 7 of our patients were investigated by spinal MR imaging and none showed spinal cord involvement, which strongly argues against spinal cord involvement as a part of the disease. The absence of spinal cord lesions in 


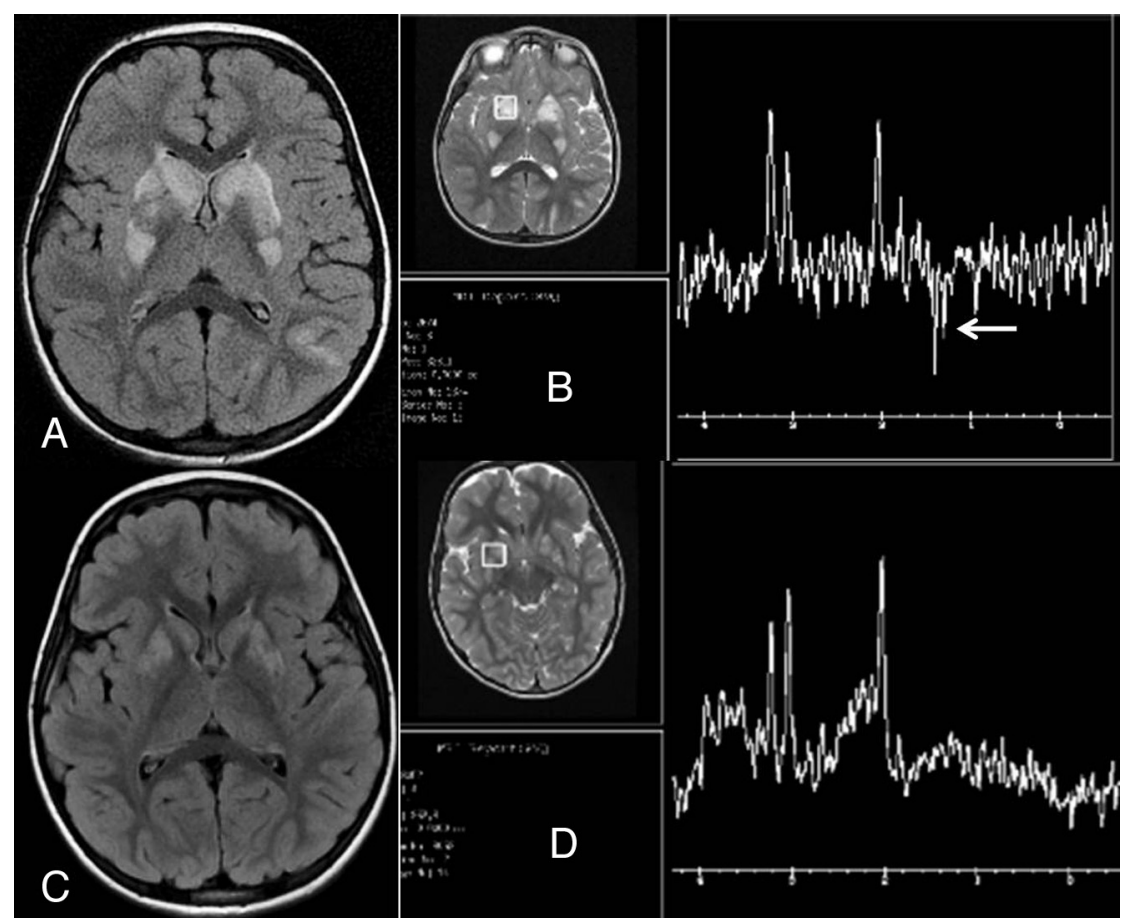

FIG 4. Female patient, 2.5 years old, with BBGD. Pretherapy axial FLAIR ( $A$ ) shows high signal and swelling of the caudate nuclei with partial involvement of the putamina. $B$, Pretherapy single voxel MR spectroscopy at the right basal ganglia at $144 \mathrm{~ms}$ shows abnormal inverted lactate doublet (arrow) indicating lactate elevation and decreased NAA peak. C, Posttherapy axial FLAIR shows regressive changes of the abnormal signal and swelling in the basal ganglia. $D$, Posttherapy single voxel MR spectroscopy at the right basal ganglia at $144 \mathrm{~ms}$ shows disappearance of the lactate doublet with subtle improvement of NAA peak.

our patients is consistent with all previously described cases, except the 1 reported by Alfadhel et al. ${ }^{7}$

Spectroscopy $\left({ }^{1} \mathrm{H}-\mathrm{MR}\right)$ was performed in 6 of our cases at time of onset of symptoms and before initiation of therapy. It depicted lactate peaks and decreased NAA in all cases. The lactate doublet could be interpreted as sign of ongoing apoptosis. It has disappeared in the single posttherapy MR spectroscopy. Decreased NAA could be explained by early brain injury and neuronal loss. ${ }^{13}$ We found no appreciable differences between the MR spectrum in basal ganglia and in parieto-occipital cortical-subcortical lesions. As increased CNS lactate can be found in other pathologic processes including mitochondrial diseases, ${ }^{14}$ these findings are considered nonspecific. ${ }^{13}$

In our study, because assessment of the response to therapy was limited clinically to the reversibility of symptoms and radiologically to the regression of the swelling and vasogenic edema of the affected regions, none of our patients had follow-up MR spectroscopy except 1. Interestingly, in this patient (Fig 4), the lactate peak has almost disappeared reflecting that MR spectroscopy could provide additional information as a monitor of response to therapy. The short half-time of lactate could be valuable as an early indicator of response to treatment in the future.

Bilateral and symmetrical basal ganglia lesions are caused by different systemic or metabolic conditions that share some findings with BBGD. However, the dramatic response to biotin therapy and persistence of caudate and putaminal signal abnormality without neurologic sequelae are characteristic features of BBGD. Wernicke encephalopathy (WE) is an example of these diseases. It can involve the medial dorsal nucleus in thalami, periventricular regions of the third ventricle, brain stem, central gray matter, basal ganglia, and cerebellum similar to BBGD. However, the main MR imaging differences between the two are that in BBGD, the mammillary bodies are spared when usually involved in WE and the supra- and infratentorial cortical involvement in BBGD is more extensive compared with WE. ${ }^{15}$

Mitochondrial disorders can also mimic BBGD because of similarity in deep gray matter involvement. They include Leigh disease, MELAS (mitochondrial myopathy, encephalopathy, lactic acidosis, and strokelike episodes), and MERRF (myoclonic epilepsy associated with ragged red fibers). Like BBGD, Leigh disease typically causes symmetric putaminal involvement, which may be associated with abnormalities of caudate nuclei, thalami, and brain stem; however, it less frequently involves the cerebral cortex and rarely involves the white matter. ${ }^{16}$ In MELAS, the multiple cortical and subcortical infarctlike lesions that cross vascular boundaries are a distinctive feature. ${ }^{16}$ In MERRF, the imaging findings are nonspecific. The cerebral and cerebellar white matter is reported to show patchy $\mathrm{T} 2$ prolongation on MR imaging. Cerebral and cerebellar atrophy are almost always present. Involvement of the deep gray matter nuclei with degeneration and calcification of dentate and globus pallidus are the most common manifestations. ${ }^{17}$ On the contrary, in BBGD the globus pallidus is usually not affected. ${ }^{1,2,13,18}$ This also differentiates the disease from toxic causes of encephalopathy such as carbon monoxide poisoning, which has propensity to affect the globus pallidus. ${ }^{19}$

Although the disease described responds to biotin, biotinidase deficiency is absent. The brain imaging findings are also different from those seen in biotinidase deficiency, in which the neuroradiologic changes indicate diffuse low attenuation of the white matter followed by progressive marked cerebral atrophy. ${ }^{20}$

In contrast to the swelling of the caudate heads and putamen in patients with BBGD, the main radiologic feature in juvenile Huntington disease is caudate atrophy and increased proton attenuation and T2 signal in the atrophic caudate nuclei and putamina. ${ }^{21}$ In patients with acute destructive lesions of the basal ganglia, ie, the so-called benign form of infantile bilateral striatal necrosis, the basal ganglia lesions might return to normal ${ }^{22}$ while they persist after treatment in BBGD. ${ }^{4}$

Generally, BBGD should be thought of in any pediatric patient with neurologic symptoms and bilateral and symmetrical affection of caudate nuclei and putamina in MR imaging. Biotin and thiamine treatment is then suggested until either BBGD is excluded or 1 of the other differential diseases has been clearly identified. ${ }^{23}$ 
In our study, 12 cases ( $80 \%$ ) had a good (favorable) outcome with no clinical sequelae, whereas 3 patients had an unfavorable outcome, 2 (13.3\%) had dystonia and dysarthria, and 1 patient $(6.7 \%)$ died from severe encephalopathy because of delayed diagnosis and treatment. These data suggested better outcome results when compared with Alfadhel et $\mathrm{al}^{7}$ who had 4 of 18 (22\%) patients who died, 6 (33.3\%) patients who showed mild to moderate neurologic deficit, and $2(11 \%)$ cases in which the patients had severe neurologic deficits.

The gap between the age of disease onset and the date of starting treatment by biotin and thiamine correlates directly with the neurologic outcome. Two of our 15 patients who had delayed diagnosis displayed neurologic deficits and another patient died for the same reason; the remaining patients who were diagnosed early and received immediate treatment achieved good outcomes and became asymptomatic.

It is important to check for the presence of BBGD in children with acute onset extrapyramidal symptoms as it can be managed without further neurologic deterioration. ${ }^{3}$ Because there is no known biochemical marker for the disease, the only way to confirm the diagnosis is the detection of a mutation in the SLC19A3 gene. ${ }^{6}$

\section{CONCLUSIONS}

BBGD is a treatable underdiagnosed condition. Children with unexplained encephalopathy and bilateral signal alterations of the caudate nucleus and putamen in MR imaging should be suspected of having the disease and a therapeutic trial with biotin and thiamine seems to be mandatory as the prognosis depends on the time interval between the diagnosis and initiation of therapy.

\section{REFERENCES}

1. Ozand PT, Gascon GG, Al Essa M, et al. Biotin-responsive basal ganglia disease: a novel entity. Brain 1998;121:1267-79

2. El-Hajj TI, Karam PE, Mikati MA. Biotin-responsive basal ganglia disease: case report and review of the literature. Neuropediatrics 2008;39:268-71

3. Bindu PS, Noone ML, Nalini A, et al. Biotin-responsive basal ganglia disease: a treatable and reversible neurological disorder of childhood. J Child Neurol 2009;24:750-52

4. Debs R, Depienne C, Rastetter A, et al. Biotin-responsive basal ganglia disease in ethnic Europeans with novel SLC19A3 mutations. Arch Neurol 2010;67:126-30

5. Yamada K, Miura K, Hara K, et al. A wide spectrum of clinical and brain MRI findings in patients with SLC19A3 mutations. BMC Med Genet 2010;11:171-79

6. Reihle C, von Kalle T, Severien C, et al. Biotin-responsive basal gan- glia disease in a 10-year-old German girl without SLC19A3 gene mutation. In: 39th Annual Meeting of the Society of Neuropediatrics, Innsbruck, Austria. April 25-28, 2013

7. Alfadhel M, Almuntashri M, Jadah RH, et al. Biotin-responsive basal ganglia disease should be renamed biotin-thiamine-responsive basal ganglia disease: a retrospective review of the clinical, radiological and molecular findings of 18 new cases. Orphanet J Rare Dis 2013;8:83-87

8. Zempleni J, Hassan YI, Wijeratne SS. Biotin and biotinidase deficiency. Expert Rev Endocrinol Metab 2008;3:715-24

9. Zeng WQ, Al-Yamani E, Acierno JS Jr., et al. Biotin-responsive basal ganglia disease maps to $2 \mathrm{q} 36.3$ and is due to mutations in SCL19A3. Am J Hum Genet 2005;77:16-26

10. Spector R, Mock D. Biotin transport through the blood-brain barrier. J Neurochem 1987;48:400-04

11. Vlasova TI, Stratton SL, Wells AM, et al. Biotin deficiency reduces expression of SLC19A3, a potential biotin transporter, in leukocytes from human blood. J Nutr 2005;135:42-47

12. Rodriguez-Melendez R, Zempleni J. Regulation of gene expression by biotin (review). J Nutr Biochem 2003;14:680-90

13. Tabarki B, Al-Shafi S, Al-Shahwan S, et al. Biotin-responsive basal ganglia disease revisited: clinical, radiologic, and genetic findings. Neurology 2013;80:261-67

14. Lin DD, Crawford TO, Barker PB. Proton MR spectroscopy in the diagnostic evaluation of suspected mitochondrial disease. AJNR Am J Neuroradiol 2003;24:33-41

15. Zuccoli G, Santa Cruz D, Bertolini M, et al. MR imaging findings in 56 patients with Wernicke encephalopathy: nonalcoholics may differ from alcoholics. AJNR Am J Neuroradiol 2009;30:171-76

16. Cheon JE, Kim IO, Hwang YS, et al. Leukodystrophy in children: a pictorial review of MR imaging features. Radiographics 2002;22:461-76

17. Barkovich AJ, Good WV, Koch TK, et al. Mitochondrial disorders: analysis of their clinical and imaging characteristics. AJNR Am J Neuroradiol 1993;14:1119-37

18. Roig M, Calopa M, Rovira A, et al. Bilateral striatal lesions in childhood. Pediatr Neurol 1993;9:349-58

19. Hegde AN, Mohan S, Lath N, et al. Differential diagnosis for bilateral abnormalities of the basal ganglia and thalamus. Radiographics 2011;31:5-30

20. Bousounis DP, Camfield PR, Wolf B. Reversal of brain atrophy with biotin treatment in biotinidase deficiency. Neuropediatrics 1993; 24:214-17

21. Ho VB, Chuang HS, Rovira MJ, et al. Juvenile Huntington disease: CT and MR features. AJNR Am J Neuroradiol 1995; 16:1405-12

22. Roig M, Macaya A, Munell F, et al. Acute neurologic dysfunction associated with destructive lesions of the basal ganglia: a benign form of infantile bilateral striatal necrosis. J Pediatr 1990;117: $578-81$

23. Distelmaier F, Huppke P, Pieperhoff P, et al. Biotin-responsive basal ganglia disease: a treatable differential diagnosis of Leigh syndrome. JIMD Rep 2014;13:53-57 